\title{
Radiologic Management of COVID-19: Preliminary Experience of the Iranian Society of Radiology COVID-19 Consultant Group (ISRCC)
}

\author{
Arash Mahdavi (iD ${ }^{1}$, Nastaran Khalili ${ }^{2}$, Amir H. Davarpanah (iD ${ }^{3}$, Taraneh Faghihi ${ }^{1}$, Ali Mahdavi (iD $^{4}$, Sara \\ Haseli (iD) ${ }^{5}$, Ali Sabri (iD ${ }^{6}$, Shahram Kahkouee ${ }^{5}$, Mohammad Ali Kazemi ${ }^{7}$, Payam Mehrian ${ }^{5}$, Farahnaz \\ Falahati ${ }^{8}$, Mehrdad Bakhshayeshkaram (iD ${ }^{5,}{ }^{*}$ and Morteza Sanei Taheri (iD ${ }^{8,9,{ }^{* *}}$ \\ ${ }^{1}$ Department of Radiology, Modarres Hospital, Shahid Beheshti University of Medical Sciences, Tehran, Iran \\ ${ }^{2}$ School of Medicine, Tehran University of Medical Sciences, Tehran, Iran \\ ${ }^{3}$ Department of Radiology and Imaging Sciences, Emory University School of Medicine, Atlanta, GA, United States \\ ${ }^{4}$ Department of Radiology, Imam Hossein Hospital, Shahid Beheshti University of Medical Sciences, Tehran, Iran \\ ${ }^{5}$ National Research Institute of Tuberculosis and Lung Diseases (NRITLD), Department of Radiology, Masih Daneshvari Hospital, Tehran, Iran \\ ${ }^{6}$ Department of Radiology, McMaster University, Niagara Health, Ontario, Canada \\ ${ }^{7}$ Department of Radiology, Amiralam Hospital, Tehran University of Medical Sciences, Tehran, Iran \\ ${ }^{8}$ Iranian Society of Radiology, Tehran, Iran \\ ${ }^{9}$ Department of Radiology, Shohada-E-Tajrish Hospital, Shahid Beheshti University of Medical Sciences, Tehran, Iran \\ "Corresponding author: Department of Radiology, Chronic Respiratory Diseases Research Center, National Research Institute of Tuberculosis and Lung Diseases (NRITLD), \\ Shahid Beheshti University of Medical Sciences, Postal Code: 1955841452, Tehran, Iran, Tel: +98-2126109590, Email: mehrdadbakhshayesh@yahoo.com \\ "Corresponding author: Department of Radiology, Shohada-E-Tajrish Hospital, Shahid Beheshti University of Medical Sciences, Postal Code: 1989934148, Tehran, Iran. Tel: \\ +98-2122701628, Email: saneim@gmail.com
}

Received 2020 March 01; Revised 2020 March 04; Accepted 2020 March 05.

Keywords: Management, Diagnostic Radiology, COVID-19

\section{Dear Editor,}

In December 2019, a novel coronavirus, now commonly known as the COVID-19, emerged in Wuhan, China, causing pneumonia and acute respiratory failure. This highly contagious virus has been spreading rapidly worldwide, with more than 50 countries being currently affected. As of February 25th, more than 2,600 cases of COVID-19 have been reported outside of China. In Iran, the number of COVID-19 positive cases raised to 245 in just a week from when the first case was initially reported. To this date, Iran has experienced the highest number of COVID-19-related deaths after China (1). Elimination of COVID-19 is a global health priority because as long as the virus continues to circulate in any region of the world, increase in infection and mortality rate is inevitable.

Coronaviruses are nonsegmented, enveloped, positivesense, single-strand ribonucleic acid viruses, belonging to the Coronaviridae family. So far, six types of coronaviruses had been identified of which only two had caused epidemics with high mortality rates; the Middle East respiratory syndrome (MERS-CoV) and the severe acute respiratory syndrome (SARS-CoV) (2). However, seven years after the MERS epidemic, the new COVID-19 virus was introduced in 2019, quickly causing a global outbreak (3). Pa- tients infected with this novel COVID-19 virus manifested with symptoms of severe pneumonia, including fever, fatigue, dry cough, and acute respiratory distress (4). Primary investigations revealed a mean incubation period of 5.2 days for the novel COVID-19-infected pneumonia (NCIP), allowing for the rapid global spread of the disease through air travelers (5). Current evidence suggests that asymptomatic patients have the ability to transmit the virus and contaminate others during their incubation period. Moreover, high sputum viral loads still exist during patient's recovery period (6). Due to these reasons, the possibility of a pandemic is not far from mind.

According to available guidelines, the diagnosis of COVID-19 should be confirmed by reverse-transcription polymerase chain reaction (RT-PCR) or gene sequencing of respiratory or blood specimens. However, the RT-PCR has a detection rate as low as $30 \%$ to $60 \%$ at initial presentation as well as being associated with several limitations (7). This delay in diagnosis and treatment subsequently results in a higher risk of infecting a larger population. Thus, finding a strategy for the early diagnosis of COVID-19 is of outmost importance.

Imaging is a critical tool in diagnosing patients suspicious for COVID-19 infection, as well as in evaluating the 
severity and disease progression in COVID-19 infection. In comparison to RT-PCR, chest CT imaging is a more reliable, feasible and rapid method to diagnose and assess COVID19 , especially in epidemic regions. In a recent report from China, chest CT-scan had a sensitivity of $97 \%$ for diagnosing COVID-19, superior to RT-PCR (8). Therefore, in addition to being aware of the imaging manifestations of the novel COVID-19 infection, it is essential for radiologists and radiologic departments across the world to implement strategies for the optimal management of this situation.

Here we summarize the current strategy of the Iranian Radiologic Consultant Group in terms of diagnosis and management of cases suspicious of COVID-19.

In February 2019, with the increasing cases of the novel COVID-19 in Iran and the fear of turning into a serious outbreak, a central committee was established on behalf of the Iranian radiology society in order to provide consultations for physicians dealing with known or suspected cases of NCIP. To reach this goal, the radiologic imaging (including both chest X-ray and chest CT scan) of all patients presenting with symptoms characteristic of NCIP are gathered from across the country and sent to this group via social media or PACS. After interpretation of the imaging features by a group of experienced radiologists from Iran, USA and Canada (ISRCC group), reports are integrated to reach a final conclusion.

To date, the Iranian Society of Radiology COVID-19 Consultant Group (ISRCC) has taken the following steps:

- Created educational videos regarding the radiologic features of NCIP based on current articles and proved cases in Iran.

These videos have been widely distributed in social media and medical-related websites, providing easy and fast access for all physicians, particularly radiologists, and have received more than 100,000 views on social networks so far. It is of note that, in order to maintain patients' privacy, their identity was removed from all X-rays and CT slides.

- Implemented a low-dose thoracic CT scan protocol for screening and/or diagnosis of patients suspected of 19COVID based on a modified version of the existing lung cancer screening CT protocol, version 5.1, originally provided by American Association of Physicists in Medicine (AAPM) (9).

This protocol was presented to radiology centers and hospitals throughout the country in order to allow for the national harmonization of imaging methods applied.

- Designed a diagnostic protocol for immediate action in case of lack of access to the COVID-19 RT-PCR diagnostic kit.

- Established a consultation response team consisting of national and international radiologists specializing in thoracic imaging.

An easily accessible social network platform was used to provide direct consultations to physicians from different medical fields. In addition, the presence of international radiologists from North America allowed for 24hour coverage of consultation.

- Defined specific criteria for reporting radiologic features of suspected patients imaging as normal, inconsistent, or highly suggestive of COVID-19 (Figure 1).

Although this primary reporting template has been effective so far for the classification of patients, it might undergo changes after more cases have been reviewed.

- Provided suggestions for complementary diagnostic/therapeutic actions based on patients radiology report

- Investigated the possibility of using a comprehensive national/regional PACS for storing and sharing imaging of patients

The primary results have shown that low-dose CT-scan, which is a widely available and relatively inexpensive imaging test in Iran, is beneficial for the diagnosis of COVID19 in suspicious symptomatic patients. In addition, the current thoracic CT scan reporting template designed by ISRCC could be advantageous for classifying patients and predicting which patients should be treated in the outpatient setting and discharged and which patients require further evaluation and monitoring, and even admission to intensive care unit (ICU), if necessary.

We believe that the following actions conducted and supervised by the ISRCC have been successful in enhancing the radiologic management of this crisis and have facilitated the diagnosis and control of COVID-19. Thus, we are delighted to share our experience with other medical societies across the world to reduce the burden of this disease.

\section{Footnotes}

Authors' Contributions: Study concept and design: Arash Mahdavi, Amir Davarpanah, Taraneh Faghihi, Ali Mahdavi, Sara Haseli, Ali Sabri, Shahram Kakouei, Mohammad Ali Kazemi, Payam Mehrian, Farahnaz Falahati, Mehrdad Bakhshayeshkaram, and Morteza Sanei Taheri; drafting of the manuscript: Arash Mahdavi and Nastaran Khalili; study supervision: Mehrdad Bakhshayeshkaram and Morteza Sanei Taheri

Conflict of Interests: There is no conflict of interests. Funding/Support: No funding has been received. 


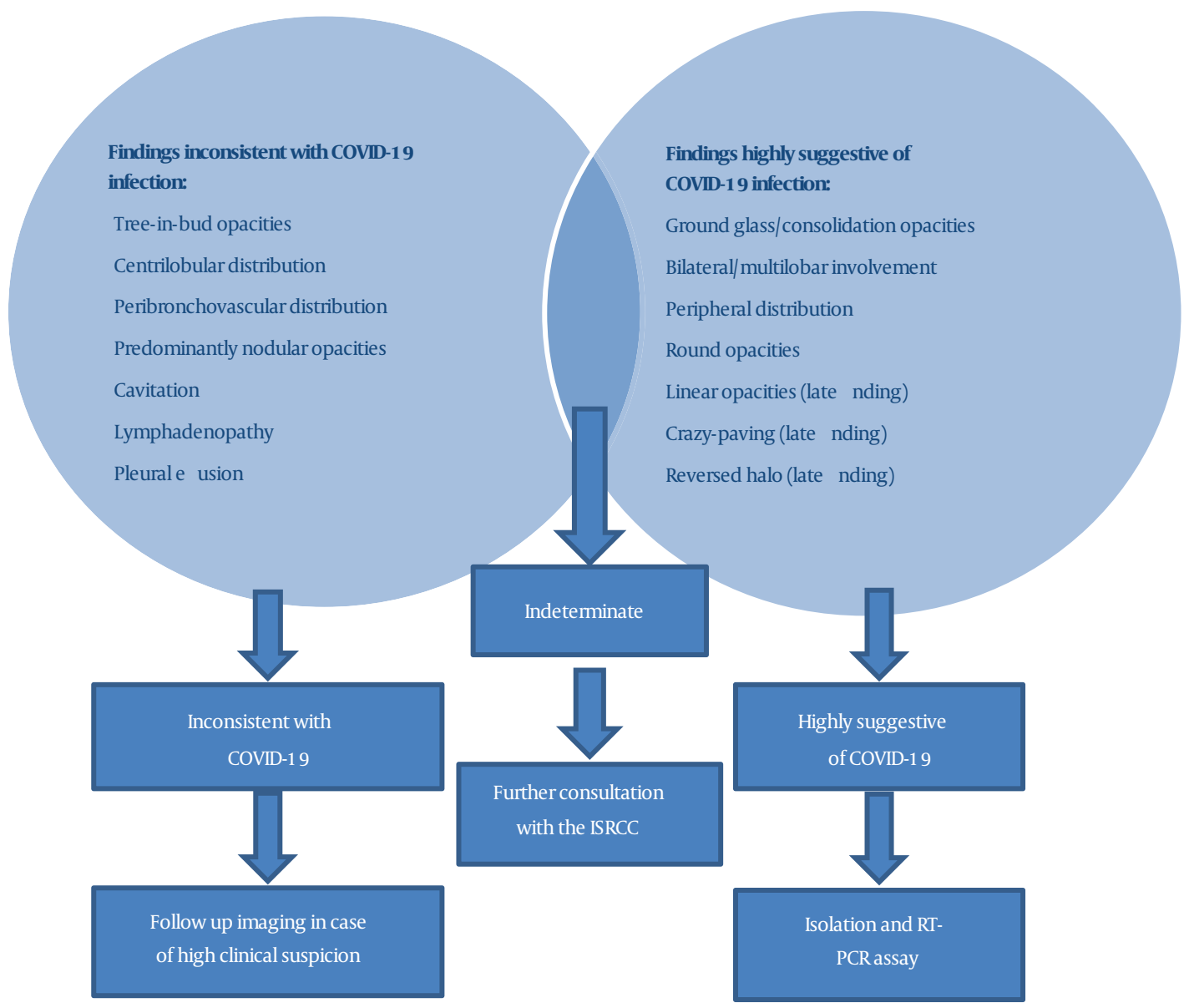

Figure 1. Reporting Template of HRCT* of patients suspicious of COVID-19 provided by the Iranian Society of Radiology COVID-19 Consultant Group (ISRCC). *High resolution CTscan: highly suggestive, presence of any highly suggestive findings without presence of inconsistent findings; inconsistent, presence of inconsistent findings; indeterminate: Presence of multiple highly suggestive findings with one or more inconsistent findings.

\section{References}

1. Johns Hopkins Center for Health Security. COVID-19. Johns Hopkins University; 2020. Available from: http://www. centerforhealthsecurity.org/resources/COVID-19/.

2. Weiss SR, Leibowitz JL. Coronavirus pathogenesis. Adv Virus Res. 2011;81:85-164. doi: 10.1016/B978-0-12-385885-6.00009-2. [PubMed: 22094080].

3. Wang C, Horby PW, Hayden FG, Gao GF. A novel coronavirus outbreak of global health concern. Lancet. 2020;395(10223):470-3. doi: 10.1016/S0140-6736(20)30185-9. [PubMed: 31986257].

4. Huang C, Wang Y, Li X, Ren L, Zhao J, Hu Y, et al. Clinical features of patients infected with 2019 novel coronavirus in Wuhan, China. Lancet. 2020;395(10223):497-506. doi: 10.1016/S0140-6736(20)30183-5. [PubMed: 31986264].

5. Li Q, Guan X, Wu P, Wang X, Zhou L, Tong Y, et al. Early transmission dynamics in wuhan, China, of novel coronavirus-infected pneumonia. $N$
Engl J Med. 2020. doi: 10.1056/NEJMoa2001316. [PubMed: 31995857].

6. Rothe C, Schunk M, Sothmann P, Bretzel G, Froeschl G, Wallrauch C, et al. Transmission of 2019-nCoV infection from an asymptomatic contact in Germany. N Engl J Med. 2020;382(10):970-1. doi: 10.1056/NEJMc2001468. [PubMed: 32003551].

7. Yang Y, Yang M, Shen C, Wang F, Yuan J, Li J, et al. Laboratory diagnosis and monitoring the viral shedding of 2019-nCoV infections. -; 2020. doi: 10.1101/2020.02.11.20021493.

8. Ai T, Yang Z, Hou H, Zhan C, Chen C, Lv W, et al. Correlation of chest CT and RT-PCR testing in coronavirus disease 2019 (COVID-19) in China: A report of 1014 cases. Radiology. 2020:200642. doi: 10.1148/radiol.2020200642. [PubMed: 32101510].

9. AAPM. Lung cancer screening CT protocols version 5.1: AAPM's working group on standardization of CT nomenclature and protocols. 2019, [cited 2019/13/9]. Available from: https://www.aapm.org/pubs/ CTProtocols/documents/LungCancerScreeningCT.pdf. 